\title{
If You Let Them Build It, They Will Stay! An Empirical Study of Add-on Content and User Engagement
}

\author{
Irfan Kanat \\ College of Business \\ Ohio University \\ Athens, Ohio
}

\author{
Kevin Hong \\ W.P. Carey School of Business \\ Arizona State University \\ Tempe, Arizona
}

\author{
Bin $\mathrm{Gu}$ \\ W.P. Carey School of Business \\ Arizona State University \\ Tempe, Arizona
}

\author{
T.S. Raghu \\ W.P. Carey School of Business \\ Arizona State University \\ Tempe, Arizona
}

\begin{abstract}
This study aims to uncover the effects of two types of add-on content -namely, user generated and developer generated content- on user engagement with software. Utilizing a novel dataset from a major online game distribution platform covering 7323 products between January 2015 and February 2016, the findings reveal that both types of add-on content increases the engagement with software. However, we observe substitutive patterns between different types of add-on content. Our results suggest integrating social features to the base product reduces these substitution effects. The results of this study contribute to the literatures on user engagement and add-on content by uncovering hitherto overlooked substitutive relations between user generated and developer generated addon content.
\end{abstract}

Keywords-Online Games; Add-on Content; User Engagement; Digital Economy;

Having new heroes to the game is kind of vital to the longterm involvement and investment that the players have. You want to feel like it's a living, breathing game that always has something new and exciting that's being added to it.

Aaron Keller, Asst. Game Director, Blizzard Interactive [1]

\section{INTRODUCTION}

Changing business models in software industry are resulting in the need for an emphasis on longer term relations and engagement with users rather than closing one time sales [2], [3]. Prominent gaming and productivity software vendors (e.g., Microsoft, Adobe, Valve) have moved to licensing models selling functionality on an on-demand basis [2], [4]. This shift in business models requires vendors to attract and capture users' continued interest, and to engage users to spend time in their system [5], [6]. Game industry is a prime context to study engagement, as the survival depends heavily on engagement [7], [8]. In hedonic contexts such as gaming, the implications are even more salient as multiple products compete for users' scarce attention. Valve software's Team Fortress 2 (TF2) is a prime example of keeping users engaged over the years. In 2014, TF2 was the second most popular game on the Steam ${ }^{1}$ seven years after its release [9], an unusual accomplishment in this dynamic

\footnotetext{
${ }^{1}$ Steam is the largest gaming software distribution platform in the world
}

market. Valve software's extraordinary success raises the question How can the developers keep their users engaged?

The key to continued engagement is add-on content. Addon content allows the firm, to provide additional features to customers who own the base product [10]. More recently, the software distribution platforms are creating opportunities to enhance game design after its initial release through distribution of add-on content in the form of community produced user generated content (UGC) ${ }^{2}$ and developer produced downloadable content $(\mathrm{DGC})^{3}$ [11], [12]. A series of carefully timed DGC and UGC releases was behind TF2's unending relevance in the gaming world, as is evidenced by Valve software paying $\$ 57$ Million to the community modders since 2011 for add-on content [13]. As Valve software CEO Gabe Newell puts it:

...the community itself makes ten times as much content as we do, we can't compete with our own customers. Our customers have defeated us, not by a little but by a lot. They're buiding content that's just as good or better than what we're building and they're building it at a spectacular rate [14].

The customer base for the add-on content however is determined by the base product. The companies through their decisions with regards to design of the base product determine the effectiveness of add-on content [15]. As contingency theory posits, firms need to adjust their strategies to the realities of their context [16]. Gaming industry is no different, in the sense that developers position their games in certain segments of the markets (social games, single player games, indie games, etc.) through fundamental design decisions. These initial design decisions such as social features or novelty differentiate the products in this hyper competitive environment, defining the game's performance going forward [17]. These segment choices -and design decisions leading up to them- should dictate subsequent

\footnotetext{
${ }^{2}$ Such as, 'Dust 2 Night' a version of famous Counter Strike map Dust that takes place at night, available for download on Steam Workshop for free.

${ }^{3}$ Like the 'Burial at Sea' adding a whole new single player scenario to the famous Bioshock Infinite on sale in Steam for \$14.99.
} 
design and add-on content decisions. Properly aligning these design decisions can increase fit between the context and firm's strategy, leading to increased engagement.

It has been demonstrated that engaged users use the systems more [5], [18], and spend more money on premium content [19]. Given the rise in the popularity of business models that rely on continued engagement [2], [3], our goal is to investigate how add-on content would impact user engagement in the context of gaming software, and to uncover strategies in aligning the design features and add-on content to increase engagement. In order to assess the business value of such strategies, it is essential to establish the effects of user and developer generated content on users' engagement. However, the market level evidence for the effect of these fundamental design choices is a nascent area IS researchers are just beginning to investigate. Our understanding of market level effects of fundamental decisions -such as whether to integrate social features, or to integrate designs of community developers- is lacking. Furthermore, we have little empirical evidence on possible complementarities between these decisions. Understanding such complementary/subsitutive relations is crucial to increase engagement level of software. In lack of such understanding, developers may miss opportunities to increase engagement through strategically combining or withholding different strategies. Therefore, this paper seeks to address the following research questions: (1) How does add-on content strategy affect user engagement? (2) Are UGC and DGC complimentary or substitutive to each other? (3) How does the base product design alter the effects of UGC and DGC?

To answer these research questions, we obtain a data set from Steam, the leading game distribution platform in the world with over 70\% market share [20] and over 125 Million active users [21]. Our data set includes 7,323 games in a 61 week period between January 2015 and February 2016. We use mixed effects models to investigate effects of game and platform design features on user engagement. Our results indicate that, social features enhance the effects of addon content. We found a substitutive relationship between UGC and DGC, which disappeared for purely multiplayer games. These results should inform decision making in selecting an add-on content strategy for software developers. Our research extends the prior literature on engagement in games [8], [22]-[24] by highlighting the role of add-on content strategy in user engagement, as well as interaction of different types of add-on content.

The remainder of the paper is structured as follows: In Section II, we survey the extant literature on user engagement and add-on strategy, and propose theoretically grounded hypotheses. Section III presents a discussion of data and the empirical models used in this study. We present the results of estimation in Section IV. Finally, this paper is concluded with a discussion of key findings and further research directions in Section V.

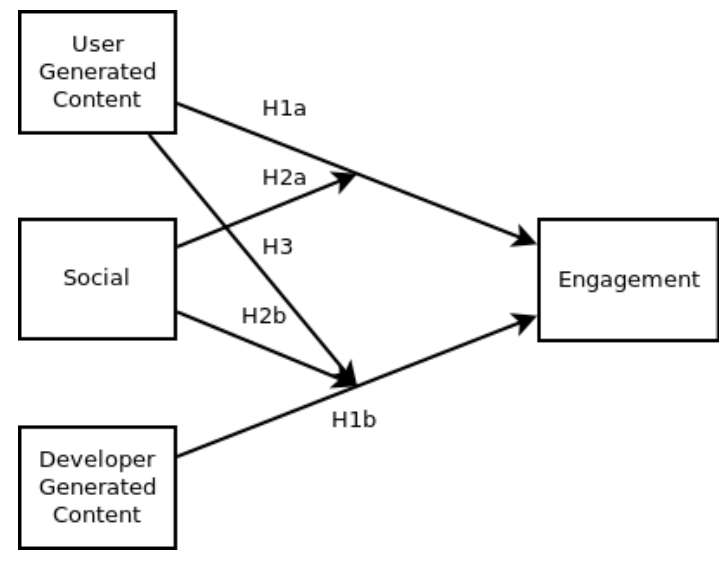

Figure 1. Hypothesized Relations

\section{Related Literature AND TheORy DeVelopment}

User engagement in the IS context carries two distinct meanings: (1) The involvement of the users in the design process [25], [26], and (2) users' experience while using the system [27], [28]. We focus on the second meaning, user engagement as the ability of the IT artifact to attract and retain user attention [5]. Prior literature has found that users' need to autonomy, competence and relatedness to drive engagement with games [5], [29], [30]. Engagement in turn, leads to increased and repeated use of the system as well as participation in community activities. It has been shown that higher engagement increases willingness to pay for premium services [19] and community engagement has been shown to increase both utilitarian and hedonistic benefits of the systems [31].

Considering the new business models that rely on continued engagement and repeated sales [2], [3], [32] extending the lifetime of the product is important for games. There are two major sources of engagement in games, (1) content provided by the developer and (2) interactions with other players [22]-[24]. Figure 1 presents an overview of investigated relationships.

The games with a single player component often provide some content (e.g. a scripted scenario) that the players can enjoy alone. In its essence, as with any information system, a game is a facilitator to acces content [33]. Digital games are hedonic information goods, like movies and books a typical user will only go through the provided content once [34]. The content provided by the game engages users by providing novelty and challenge [5], [35]. Once a user goes through the content provided by a game the novelty and challenge provided quickly diminishes, and players move on to other games. The single player component is more similar to a book or a movie, generally speaking, this part is only engaging for a while. Once a user exhausts the available single player content, the engagement is over.

More recently, add-on content emerged as a way to lend 
an existing product a new lease on life in this context [12], [36]-[38]. These add-ons allow new content to be added to the game after release. The add-on products in the software context sits somewhere between updates and versioning [3], [39]-[41]. Unlike updates, not every customer receives the add-on content, and unlike versioning, the features are not bundled into different versions but are sold or given away separately. A customer can purchase the base product and enhance its capabilities any time through these add-on products [10], [42]. Unfortunately literature on effects on Add-on content on engagement has been scarce.

There are two broad strategies when it comes to providing add-on content. First is for the developers to provide the add-on content (consider SAS), this is referred to in the gaming circles as Downloadable Content (DLC) [12], [36]. The other is to get the community to provide the add-on content (consider R), which is often referred to as User Generated Content (UGC) [37], [38], [43], [44]. Introducing new content will introduce novelty and new challenges, hence keeping users motivated to keep using the system. Hence we anticipate both forms of add-on content to increase engagement.

Hypothesis 1a: User generated content will increase engagement with the software.

Hypothesis 1b: Developer generated content will increase engagement with the software.

The second way a game keeps users engaged is the player to player interactions. The effect of player to player interactions is two folds, first it satisfies the users' need for relatedness [29], [30]. Second, unlike scripted interactions the single player component provides, player to player interaction can evolve in unscripted, dynamic ways hence provide a different experience each time a game is played [8], [22], satisfying the users' social and novelty seeking needs [5], [30], [45]. We posit that the dynamic nature of player to player interactions serve as a multiplier for a content's engagement. The dynamic nature of player to player interaction will keep the content that facilitates such engagement engaging for a longer time compared to scripted interactions a single player experience provides. In the context of add-on products this means social features that enable multiplayer interactions will enhance the effect of add-on content.

Hypothesis 2a: The social features will enhance the effects of user generated content.

Hypothesis $2 b$ : The social features will enhance the effects of developer generated content.

While the developers can and do provide both types of add-on content, we do not know if the add-on strategies are substitutive or complementary to one another. Having two different channels for content can either garner competition, or synergy. The literature on multi-channel competition in content access has found conflicting results. On the one hand there is the channel competition view, whereby one channel displaces the other [46], [47]. One explanation of this displacement phenomenon comes from search costs, whereby the search costs for users to find out about add-on content leads to competition effects [48]. Having two competing channels for add-on content will mean the users may end up using one or the other [49]. On the other hand there is the channel complementarities view [50], [51]. Users' choice of channels is a function of user preferences [52]. Based on the different types of content served by alternative channels, media complementarity theory posits that users utilize one channel to expand upon the content they found in another [50]. This cross utilization of different channels to access specific types of content creates complementary effects. The literature is far from conclusive, especially when it comes to add-on content in software markets. We build our hypotheses on the long established search costs theory. Hence the following:

Hypothesis 3: There will be substitution effects between UGC and DGC.

The literature is silent on the role of social features in add-on channel preferences. We use search costs theory to explain this relationship. As argued above, having two competing channels, will induce learning costs on users. The users will reduce their costs by predominantly using one channel. Literature highlights the role of social learning as a mechanism to reduce search costs [53], [54]. When a user can observe others' behavior, she can learn from their experience [55], [56]. The user to user interactions afforded by social features allow users to observe each others' add-on preferences. Reducing the search costs of finding out about add-on content. Hence we propose:

Hypothesis 4: The substitution effect will be more significant for single player games.

In this study we investigate the role of add-on strategy on user engagement. To the best of our knowledge, this is an area of IS research that has not yet been investigated. While it is true that there are related work on software updates and versioning, our study presents the first empirical work on the effects of add-on content on user engagement.

\section{EMPIRICAL Methods}

\section{A. Data and Variables}

Our data comes from Steam, the largest online software distribution platform with over 125 Million active users [21] and over $70 \%$ market share for downloadable games [20]. We wrote python scripts to collect publicly available data from Steam API, product pages and user profiles. The scripts were deployed on an Amazon AWS EC2 server and ran daily. The weekly aggregated data covers a 61 week period between Jan, 1, 2015 to Feb, 25, 2016. The dataset tracks 7323 products and has 398201 observations. Table I presents demographics for our variables.

To derive our Engagement and Percent metrics, we randomly sampled around 185.000 users every day and recorded 
Table I

DESCRIPTIVE STATISTICS

\begin{tabular}{|c|c|c|c|c|c|c|c|c|c|c|c|c|c|}
\hline & mean & sd & AvgMin & Users & Percent & multi & New & Price & Discount & free & Bundled & Uniq & UGC \\
\hline AvgMin & 235.03 & 723.94 & & & & & & & & & & & \\
\hline Users & 421.00 & 12820.63 & $0.34^{* * *}$ & & & & & & & & & & \\
\hline Percent & 0.00 & 0.00 & $0.36^{* * *}$ & $0.61 * * *$ & & & & & & & & & \\
\hline Social & 0.36 & 0.48 & $0.08^{* * * *}$ & $0.03 * * *$ & $0.09 * * *$ & & & & & & & & \\
\hline New & 0.03 & 0.17 & $0.01 * * *$ & $0.01 * * *$ & $-0.03 * * *$ & $-0.01 * * *$ & & & & & & & \\
\hline Price & 11.87 & 13.51 & $0.20 * * *$ & $0.00 *$ & $0.04 * * *$ & $0.04 * * *$ & $0.04 * * *$ & & & & & & \\
\hline Discount & 0.06 & 0.15 & $-0.01 * * *$ & $-0.01 * *$ & $0.01 * * *$ & $-0.03 * * *$ & $-0.03^{* * *}$ & $0.01 * * *$ & & & & & \\
\hline free & 0.04 & 0.19 & $0.08 * * *$ & $0.08^{* * *}$ & $0.15^{* * *}$ & $0.13^{* * *}$ & $0.06 * * * *$ & $-0.17 * * *$ & $-0.07 * * *$ & & & & \\
\hline Bundled & 0.35 & 0.48 & $-0.04 * * *$ & $0.01 * * *$ & $0.07 * * *$ & $-0.12 * * *$ & $-0.09 * * *$ & $-0.10^{* * * *}$ & $0.11^{* * *}$ & $-0.14 * * *$ & & & \\
\hline Uniq & 0.01 & 0.01 & $0.14 * * *$ & $0.01 * * *$ & $0.08^{* * *}$ & $-0.09 * * *$ & $0.01 * * *$ & $0.20 * * *$ & 0.00 & $0.01 * * *$ & $0.05^{* * *}$ & & \\
\hline UGC & 194.15 & 8664.42 & $0.11^{* * *}$ & $0.08 * * *$ & $0.16^{* * * *}$ & $0.02 * * *$ & 0.00 & $0.01 * * *$ & 0.00 & 0.00 & $0.02 * * *$ & 0.00 & \\
\hline DGC & 0.24 & 0.72 & $0.24 * * *$ & $0.07 * * *$ & $0.20^{* * *}$ & $0.10^{* * *}$ & $0.01 * * * *$ & $0.17 * * *$ & $0.02 * * *$ & $0.18^{* * *}$ & $0.05^{* * *}$ & $0.10^{* * *}$ & $0.00 * *$ \\
\hline
\end{tabular}

their ownership and play times up to the point of observation. We carried out power calculations and determined a sample size that limited the margin of error to .002 at $.05 \alpha$ level.

Below is a brief discussion of variables used in this study.

Engagement: We used average minutes played as a measure of engagement. The variable was highly skewed, in the models discussed below we used log transformed average minutes played as the dependent variable.

Percent: Percent is the percentage of users in the sample that owned a given game. It is a measure of user base. Since this is a long tail market, most games have less than 1 percent market share.

Social: An indicator variable for multi player features in the game. If the game has social features this variable is set to 1,0 otherwise.

New: An indicator variable for games in their first two months of observation.

ListPrice: Daily recommended sale price for the game. Newer games and games with more content usually sell for higher prices.

Discount: Daily discount ratio, calculated over the list price. We used one lag to integrate previous days' observation.

Bundled: If a game has been part of a bundle of deeply discounted games before. Set to 1 after bundling period, 0 otherwise.

User Generated Content (UGC): Number of user generated additional content items for the game. We used one lag to integrate previous days' observation.

Developer Generated Content (DGC): Number of developer generated content for the game. We used one lag to integrate previous days' observation.

Novelty: Each game is tagged by the users based on their features (i.e: action, great soundtrack, atmospheric, etc.). To construct a meaningful measure of game novelty, we collected up to 20 most popular user generated tags for each game. We weighted each tag inversely proportional to the frequency they appeared across the games and adjusted for the total number of tags a game received. The higher scores on this variable mean that the game has more novel features. See Equation 1 to see how we calculated this variable. $\mathbf{T}$ is the tag (i) - game (j) matrix, the $v$ is the tag weights vector, $s$ is the novelty score vector for games. For example let us say a game has two tags, Action and Poetry. Action is very common, appearing in 250 games, and Poetry is a rare tag, appearing in two games. The novelty score for this game would be calculated as follows: $S=\frac{1 / 250+1 / 2}{2}=0.252$

$$
\mathbf{T}(i \times j), \quad v=\frac{1}{1^{\prime} \mathbf{T}} \quad \text { and } \quad s=\frac{\mathbf{T} v^{\prime}}{\mathbf{T} 1}
$$

\section{B. Estimation Model}

Games are hedonistic goods and as such each has to differentiate itself from the competition. This is also reflected for add-on content developed for the games [37]. To control for this game level heterogeneity, we choose a mixed effects model with random intercepts at game level and random coefficients at add-on content level. Each game will have a diffferent intercept for engagement and the slopes of add-on content will vary between games. An additional benefit of this approach was that it allowed us to use both time variant and time invariant variables at the same time.

We used a mixed effects model with both random and fixed effects. Equation 2 shows the mixed effects model formula. Where $\mathbf{Y}_{i}$ is the vector of engagement variables for game $i, \mathbf{X}_{i}$ is the matrix of predictor variables of game $i$ and $\boldsymbol{\beta}$ is the fixed effects vector. $\mathbf{b}_{i}$ is the random effects vector for game $i$ and $\mathbf{Z}_{i}$ the random effects design matrix for the same.

$$
\mathbf{Y}_{i}=\mathbf{X}_{i} \boldsymbol{\beta}+\mathbf{Z}_{i} \mathbf{b}_{i}+\varepsilon_{i}
$$

In our specific example, the $\mathbf{Z}_{i}$ design matrix is created with game id and UGC, DGC in games. This means, each game has a different intercept and a different slope on the UGC and DGC variables. All independent variables are included in $\mathbf{X}_{i}$ 


\section{Results}

We integrated game and item within game random effects and ran an $\mathrm{F}$ test, the test results indicated that each random effect improved model fit. We also controlled for effects of time through weekly dummy variables. This is especially important as our dataset covers important seasonal events like Christmas and New Year holidays; combined with deep discounts, gaming activity increases in these periods. We conducted model comparisons on Maximum Likelihood estimates and each model was an improvement over the previous model. While Restricted Maximum Likelihood (REML) estimates do not lend themselves readily to model comparison, they are known to be more accurate in estimating random effects [57]. Table II shows the REML estimates for the model. We started with a baseline model with just the control variables, then integrated main effects and interactions respectively. Each model represents a significant improvement over the previous model according to $\mathrm{F}$ tests on ML estimates. Results are qualitatively consistent across models, thus we will present results from Model 3.

Control variables tell a plausible story. New games were played less in their first two months. Interestingly we observe that games with large userbases were less engaging, the games that have larger user bases are used less. This is an unexpected result as one would expect the user base to increase the engagement overall through network effects. We believe this negative effect is due to promotions and impulse buying. It has been observed that $26 \%$ of the games purchased on Steam have not been played at all [9]. Steam is well known for deals and promotions, we believe this may be triggering impulse buying of games that the players never play. This idea is partially supported by the result that discounts reduce engagement. When a game is discounted, a lot of new players will enter the user base with low average usage durations. Hence it is normal to observe this effect.

The positive and significant coefficients for UGC and DGC indicates that our Hypotheses $1 \mathrm{a}$ and $1 \mathrm{~b}$ are supported. Using Model 2 to abstract away interaction effects, we can see that a $1 \%$ increase in UGC leads to $.29 \%$ increase in average minutes used. A $1 \%$ increase in DGC on the other hand leads to a $2 \%$ increase in average minutes used.

The interaction terms of social with UGC and DGC are also significant and positive, lending support to our Hypotheses $2 \mathrm{a}$ and $2 \mathrm{~b}$. The effect of a $1 \%$ increase in single player games was an increase of $.28 \%$ in usage, whereas the same had an effect of $.40 \%$ increase in games with social features. For a $1 \%$ increase in DGC, the single player games saw an increase of $1.75 \%$ in usage, while games with social features enjoyed an increase of $2.43 \%$.

The evidence of substitution can be seen in the significant and negative interaction between DGC and UGC, supporting our Hypothesis 3. Testing Hypothesis 4 proved to be harder. The binary variable social indicates existence of social features. This means between the purely single player games and purely social games, there lies a vast gray area of games that support both modes. Tweezing out the three way interaction is harder due to this vast inbetween space. Hence we conducted a split sample analysis of different game modes. The alternative of integrating more dummies would have inflated the number of effects to be estimated significantly. See Table III for analysis of split sample analysis. Results lend support to Hypothesis 4.

While not strictly hypothesized in this paper, we see that novelty of the base game enhances the effect of UGC but not DGC. An interesting finding in its own right, that merits further inquiry.

\section{Discussion}

In this study, we addressed a significant gap in the literature by our investigation of the role of add-on content on user engagement. To the best of our knowledge, our study represents the first study to shed light into this relationship. Considering the increasing importance of maintaining user engagement for the developers' bottom-line [5], [18] our results are very relevant. To conduct our inquiry, we gathered data from a a major software distribution platform with over 125 Million active users. This gives us a unique dataset, best suited to investigate user engagement for hedonistic software products.

Another key contribution of our study is its implications for game design theory. Prior work has focused on a game's design features and their relations with engagement or enjoyment [7], [8], [22]. Our study shows the importance of the design of future add-ons for games' continued engagement. We hope our findings will guide future research into game design and engagement.

Our finding of substitution effects between UGC and DGC in single player games means the developers should consider the most effective strategy depending on their base product. Mismanaged, offering both types of add-on content can cannibalize the developers' paid add-on content and wipe away any benefits from UGC. We recommend offering both UGC and DGC for purely social games, as the substitutive relationship disappears in such cases.

The developers have a choice in developing novel features or remaining mainstream [58]. Our results indicate novel features enhance the effects of user generated content. This can be leveraged by developers with innovative base products, to extend the engagement level of their products.

The findings presented here can be expanded by future research. In this study we present an overall view of the gaming market. It is well known that the software markets exhibit characteristics of a long tail market [59], [60]. It is highly probable that add-on content may have different effects in the head of the market, compared to the tail of the market. We encourage future research into investigating the add-on strategy in head versus tail of the software markets. 
Table II

Results of MiXed EFFects Models

\begin{tabular}{|c|c|c|c|}
\hline & Model 1 & Model 2 & Model 3 \\
\hline (Intercept) & $1.9171(0.2086)^{* * *}$ & $1.8890(0.2076)^{* * *}$ & $1.9927(0.2080)^{* * *}$ \\
\hline Weekly Dummies & $Y E S$ & $Y E S$ & $Y E S$ \\
\hline New & $-0.2283(0.0055)^{* * *}$ & $-0.2244(0.0055)^{* * *}$ & $-0.2244(0.0055)^{* * *}$ \\
\hline $\log \left(\right.$ ListPrice $\left._{t-1}+1\right)$ & $0.0002(0.0081)$ & $0.0043(0.0082)$ & $0.0050(0.0082)$ \\
\hline free & $1.8375(0.1445)^{* * *}$ & $0.9243(0.1099)^{* * *}$ & $0.8209(0.1087)^{* * *}$ \\
\hline Discount $_{t-1}$ & $-0.0304(0.0068)^{* * *}$ & $-0.0223(0.0068)^{* *}$ & $-0.0222(0.0068)^{* *}$ \\
\hline Bundled & $-0.0895(0.0055)^{* * *}$ & $-0.0852(0.0055)^{* * *}$ & $-0.0853(0.0055)^{* * *}$ \\
\hline $\log \left(U G C_{t-1}+1\right)$ & & $0.2892(0.0124)^{* * *}$ & $0.2757(0.0174)^{* * *}$ \\
\hline $\log \left(D G C_{t-1}+1\right)$ & & $2.0027(0.0465)^{* * *}$ & $1.7503(0.0606)^{* * *}$ \\
\hline Uniq & & $0.5313(0.2794)$ & $0.4468(0.2935)$ \\
\hline $\log \left(\operatorname{Percent}_{t-1}+1\right)$ & & $-36.0983(0.8634)^{* * *}$ & $-38.5872(1.8380)^{* * *}$ \\
\hline Social & & $-0.3045(0.0533)^{* * *}$ & $-0.6081(0.0640)^{* * *}$ \\
\hline Social $\times \log \left(U G C_{t-1}+1\right)$ & & & $0.1287(0.0233)^{* * *}$ \\
\hline Social $\times \log \left(D G C_{t-1}+1\right)$ & & & $0.6784(0.0908)^{* * *}$ \\
\hline Social $\times \log \left(\right.$ Percent $\left._{t-1}+1\right)$ & & & $3.1954(2.0796)$ \\
\hline $\log \left(D G C_{t-1}+1\right) \times \log \left(U G C_{t-1}+1\right)$ & & & $-0.1290(0.0214)^{* * *}$ \\
\hline $\log \left(U G C_{t-1}+1\right) \times U n i q$ & & & $1.0406(0.3981)^{* *}$ \\
\hline $\log \left(D G C_{t-1}+1\right) \times U n i q$ & & & $-0.2298(0.9372)$ \\
\hline Variance: AppID.(Intercept) & 6.0002 & 6.0608 & 6.0218 \\
\hline Variance: AppID. $\log (\mathrm{UGC}+1)$ & 0.0428 & 0.0624 & 0.0709 \\
\hline Variance: AppID. $\log (\mathrm{DGC}+1)$ & 0.9180 & 3.9822 & 3.8295 \\
\hline Variance: Residual & 0.2076 & 0.2039 & 0.2039 \\
\hline AIC & 534574.0258 & 519821.3833 & 519726.6999 \\
\hline $\mathrm{BIC}$ & 535365.9762 & 520666.4607 & 520636.7834 \\
\hline Log Likelihood & -267214.0129 & -259832.6916 & -259779.3500 \\
\hline
\end{tabular}

Table III

SPLIT SAMPLE ANALYSIS

\begin{tabular}{|c|c|c|c|}
\hline & Pure Single & Pure Social & Both Modes \\
\hline (Intercept) & $2.61(0.28)^{* * *}$ & $2.42(0.15)^{* * *}$ & $2.81(0.07)^{* * *}$ \\
\hline Weekly Dummies & $Y E S$ & $Y E S$ & $Y E S$ \\
\hline New & $-0.21(0.01)^{* * *}$ & $-0.23(0.02)^{* * *}$ & $-0.22(0.01)^{* * *}$ \\
\hline $\log \left(\right.$ ListPrice $\left._{t-1}+1\right)$ & $0.05(0.01)^{* * *}$ & $-0.10(0.02)^{* * *}$ & $-0.04(0.01)^{* *}$ \\
\hline free & $0.15(0.19)$ & $2.84(0.26)^{* * *}$ & $0.76(0.21)^{* * *}$ \\
\hline Discount $_{t-1}$ & $-0.04(0.01)^{* * *}$ & $0.02(0.03)$ & $-0.01(0.01)$ \\
\hline Bundled & $-0.08(0.01)^{* * *}$ & $-0.04(0.02)$ & $-0.10(0.01)^{* * *}$ \\
\hline $\log \left(U G C_{t-1}+1\right)$ & $0.26(0.02)^{* * *}$ & $0.50(0.05)^{* * *}$ & $0.40(0.02)^{* * *}$ \\
\hline $\log \left(D G C_{t-1}+1\right)$ & $1.88(0.06)^{* * *}$ & $0.45(0.09)^{* * *}$ & $2.33(0.08)^{* * *}$ \\
\hline Uniq & $0.16(0.34)$ & $-5.44(2.72)^{*}$ & $5.21(0.71)^{* * *}$ \\
\hline $\log \left(\right.$ Percent $\left._{t-1}+1\right)$ & $-38.57(1.96)^{* * *}$ & $-18.69(2.57)^{* * *}$ & $-36.99(0.80)^{* * *}$ \\
\hline $\log \left(U G C_{t-1}+1\right) \times U n i q$ & $0.65(0.50)$ & $4.45(1.94)^{*}$ & $1.07(0.65)$ \\
\hline $\log \left(D G C_{t-1}+1\right) \times U n i q$ & $1.35(1.52)$ & $-0.99(4.39)$ & $-7.65(1.20)^{* * *}$ \\
\hline $\log \left(D G C_{t-1}+1\right) \times \log \left(U G C_{t-1}+1\right)$ & $-0.16(0.03)^{* * *}$ & $-0.03(0.04)$ & $-0.11(0.03)^{* * *}$ \\
\hline Var: AppID (Intercept) & 5.08 & 5.70 & 7.58 \\
\hline Var: AppID $\log (\mathrm{UGC}+1)$ & 0.06 & 0.09 & 0.09 \\
\hline Var: AppID $\log (\mathrm{DGC}+1)$ & 4.89 & 0.20 & 3.11 \\
\hline Var: Residual & 0.23 & 0.12 & 0.12 \\
\hline AIC & 342965.68 & 18401.18 & 104545.98 \\
\hline $\mathrm{BIC}$ & 343792.08 & 19028.15 & 105308.38 \\
\hline Log Likelihood & -171402.84 & -9121.59 & -52193.99 \\
\hline Num. obs. & 226397 & 20668 & 114762 \\
\hline Num. groups: AppID & 4223 & 395 & 2106 \\
\hline
\end{tabular}


Table IV

STATUS OF HYPOTHESES

\begin{tabular}{llc}
\hline \multicolumn{2}{l}{ Hypothesis } & Status \\
\hline \hline H1a & UGC increases engagement & $\checkmark$ \\
H1b & DGC increases engagement & $\checkmark$ \\
H2a & Social features enhance effect of UGC. & $\checkmark$ \\
H2b & Social features enhance effect of DGC. & $\checkmark$ \\
H3 & There is a substitution effect between UGC and DGC. & $\checkmark$ \\
H4 & The substitution effect is less severe for social games & $\checkmark$ \\
\hline
\end{tabular}

Our results uncovered novelty had different implications for the effectiveness of different types of add-on strategy. While this relation is out of the scope of this study, uncovering the drivers of this relation would contribute to literature on innovation in product design. The add-on content allows altering product design post release and implications for increasing, or decreasing novelty of the product post release is exciting. Another area of further inquiry may be the investigation of add-on strategy from individual customer perspective. All results presented in this paper are built on market level data, collected at the product level. User level study may prove to be more precise and can reveal intricacies of add-on content effects overlooked by a product level approach. Finally, the results presented here should be checked against endogeneity, our results do not establish causal directionality.

In this study we investigated the effect of two different add-on content strategies on user engagement, a hitherto overlooked dimension of user engagement. We contribute to the literature on content consumption in presence of alternative channels. While the literature is far from settled, our results revealed substitutive patterns between different types of add-on content, lending support to media competition view of content consumption. We hope this research will pave the way for future research in add-on strategy in online software markets.

\section{REFERENCES}

[1] K. Orland, "When it's done: How blizzard dragged overwatch across the launch threshold." http://arstechnica.com/gaming/2016/05/when-its-doneoverwatchs-director-on-the-games-finished-yet-fluid-launch/, 2016. Accessed: 2016-05-25.

[2] J. Ramaprasad and R. Rapna, "Monetizing freemium communities: Does paying for premium increase social engagement?," Working paper, 2015.

[3] M. Niculescu and D. Wu, "When Should Software Firms Commercialize New Products via Freemium Business Models?," in Workshop on Information Systems and Economics, St. Louis, 2010.
[4] M. P. Papazoglou, "Service-oriented computing: Concepts, characteristics and directions," in Proceedings of the Fourth International Conference on Web Information Systems Engineering, (WISE) 2003., pp. 3-12, IEEE, 2003.

[5] H. L. O'Brien and E. G. Toms, "What is user engagement? a conceptual framework for defining user engagement with technology," Journal of the American Society for Information Science and Technology, vol. 59, no. 6, pp. 938-955, 2008.

[6] Amazon Inc., “Amazon underground." http://developer. amazon.com/underground, 2015. Accessed: 2015-09-01.

[7] J. Chen, "Flow in games (and everything else)," Communications of the ACM, vol. 50, no. 4, pp. 31-34, 2007.

[8] D. Liu, X. Li, and R. Santhanam, "Digital games and beyond: What happens when players compete.," Mis Quarterly, vol. 37, no. 1, pp. 111-124, 2013.

[9] K. Orland, "Steam gauge: Addressing your questions and concerns." http://arstechnica.com/gaming/2014/04/ steam-gauge-addressing-your-questions-and-concerns, April 2014. Accessed: 2015-04-15.

[10] S. Erat and S. Bhaskaran, "Consumer Mental Accounts and Implications to Selling Base Products and Add-ons," Marketing Science, vol. 31, no. 5, pp. 801-818, 2012.

[11] J. Juul, A casual revolution: Reinventing video games and their players. MIT press, 2010.

[12] H. Tyni and O. Sotamaa, "Extended or exhausted: how console dlc keeps the player on the rail," in Proceedings of the 15th International Academic MindTrek Conference: Envisioning Future Media Environments, pp. 311-313, ACM, 2011.

[13] A. O'Connor, "Over $\$ 57$ million paid out to steam workshop creators." http://www.rockpapershotgun.com/2015/01/ 30/steam-workshop-57-million-dollars/, January 2015. Accessed: 2015-04-19.

[14] A. Goldfarb, "Dice: Gabe newell discusses how to reinvent gaming." http://www.ign.com/articles/2013/02/07/ gabe-newell-discusses-how-to-reinvent-gaming, 2013. Accessed: 2016-04-12.

[15] V. Mahajan and R. A. Peterson, "Innovation diffusion in a dynamic potential adopter population," Management Science, vol. 24, no. 15, pp. 1589-1597, 1978.

[16] L. Donaldson, The contingency theory of organizations. Sage, 2001.

[17] M. Eisenman, "Understanding aesthetic innovation in the context of technological evolution," Academy of Management Review, vol. 38, no. 3, pp. 332-351, 2013.

[18] J. Webster and J. S. Ahuja, "Enhancing the design of web navigation systems: The influence of user disorientation on engagement and performance," Mis Quarterly, vol. 30, no. 3, pp. 661-678, 2006. 
[19] G. Oestreicher-Singer and L. Zalmanson, "Content or community? a digital business strategy for content providers in the social age," MIS Quarterly, vol. 37, no. 2, pp. 591-616, 2013.

[20] O. Chiang, "The master of online mayhem." http://www.forbes.com/forbes/2011/0228/ technology-gabe-newell-videogames-valve-online-mayhem. html, September 2011. Accessed: 2015-04-15].

[21] S. Sherif, "Steam has over 125 million active users, 8.9m concurrent peak." http://www.vg247.com/2015/02/24/ steam-has-over-125-million-active-users-8-9m-concurrent-peak/, February 2015. Accessed: 2015-04-15.

[22] F. Alemi, "An avatar's day in court: A proposal for obtaining relief and resolving disputes in virtual world games," UCLA JL \& Tech., vol. 2007, pp. 6-6, 2007.

[23] C. Roquilly, "Control Over Virtual Worlds by Game Companies: Issues and Recommendations," MIS Quarterly, vol. 35, no. 3 , pp. $653-671,2011$.

[24] N. Yee, "Motivations for play in online games," CyberPsychology \& behavior, vol. 9, no. 6, pp. 772-775, 2006.

[25] M. J. Salvo, "Ethics of engagement: User-centered design and rhetorical methodology," Technical Communication Quarterly, vol. 10, no. 3, pp. 273-290, 2001.

[26] M. I. Hwang and R. G. Thorn, "The effect of user engagement on system success: A meta-analytical integration of research findings," Information \& Management, vol. 35, no. 4, pp. 229-236, 1999.

[27] A. Sutcliffe, "Designing for user engagement: Aesthetic and attractive user interfaces," Synthesis lectures on humancentered informatics, vol. 2, no. 1, pp. 1-55, 2009.

[28] M. D. Dickey, "Engaging by design: How engagement strategies in popular computer and video games can inform instructional design," Educational Technology Research and Development, vol. 53, no. 2, pp. 67-83, 2005.

[29] R. M. Ryan, C. S. Rigby, and A. Przybylski, "The Motivational Pull of Video Games: A Self-Determination Theory Approach," Motivation and Emotion, vol. 30, pp. 344-360, nov 2006.

[30] R. M. Ryan and E. L. Deci, "Self-determination theory and the facilitation of intrinsic motivation, social development, and well-being.," American psychologist, vol. 55, no. 1, p. 68, 2000 .

[31] C. Grange and I. Benbasat, "Explaining customers utilitarian and hedonic perceptions in the context of product search within social network-enabled shopping websites," in $S I G$ HCI 2014 Proceeding, 2014. Paper 11.

[32] B. Zhang, R. Geng, and X. Chen, "Social networks in online games: The impact of peer influences on repeat purchase," in Workshop on Information Technologies and Systems, (Dallas, TX), 2015
[33] E. G. Toms, "Information interaction: Providing a framework for information architecture," Journal of the American Society for Information Science and Technology, vol. 53, no. 10, pp. 855-862, 2002.

[34] P. Nelson, "Information and consumer behavior," The Journal of Political Economy, vol. 78, no. 2, pp. 311-329, 1970.

[35] C. Fabricatore, M. Nussbaum, and R. Rosas, "Playability in Action Videogames: A Qualitative Design Model," HumanComputer Interaction, vol. 17, no. 4, pp. 311-368, 2002.

[36] R. Lizardi, "Dlc: Perpetual commodification of the video game.," Democratic Communiqué, vol. 25, no. 1, 2012.

[37] H. Postigo, "Of mods and modders chasing down the value of fan-based digital game modifications," Games and Culture, vol. 2, no. 4, pp. 300-313, 2007.

[38] O. Sotamaa, "When the game is not enough: Motivations and practices among computer game modding culture," Games and Culture, vol. 5, pp. 239-255, 2010.

[39] G. Lee and T. Raghu, "Determinants of mobile apps' success: Evidence from the app store market," Journal of Management Information Systems, vol. 31, no. 2, pp. 133-170, 2014.

[40] J. Claussen, T. Kretschmer, and P. Mayrhofer, "The effects of rewarding user engagement: The case of facebook apps," Information Systems Research, vol. 24, no. 1, pp. 186-200, 2013.

[41] Z. Jiang, "How to give away software with successive versions," Decision Support Systems, vol. 49, pp. 430-441, nov 2010.

[42] G. Ellison, "A Model of Add-on Pricing," The Quarterly Journal of Economics, vol. 120, no. 2, pp. 585-637, 2005.

[43] L. B. Jeppesen and L. Frederiksen, "Why Do Users Contribute to Firm-Hosted User Communities? The Case of ComputerControlled Music Instruments," Organization Science, vol. 17, no. July 2015, pp. 45-63, 2006.

[44] G. Cheliotis, "From open source to open content: Organization, licensing and decision processes in open cultural production," Decision Support Systems, vol. 47, pp. 229-244, jun 2009

[45] D. Weibel, B. Wissmath, S. Habegger, Y. Steiner, and R. Groner, "Playing online games against computer vs. human-controlled opponents: Effects on presence, flow, and enjoyment," Computers in Human Behavior, vol. 24, no. 5, pp. 2274-2291, 2008.

[46] S. Coffey and H. Stipp, "The interactions between computer and television usage," Journal of advertising research, vol. 37, no. 2, pp. 61-68, 1997.

[47] J. M. Kayany and P. Yelsma, "Displacement effects of online media in the socio-technical contexts of households," Journal of Broadcasting \& Electronic Media, vol. 44, no. 2, pp. 215229, 2000. 
[48] Y. Bakos, "The emerging role of electronic marketplaces on the Internet," Communications of the ACM, vol. 41, pp. 3542, aug 1998.

[49] A. P. Hardy, "The selection of channels when seeking information: Cost/benefit vs least-effort," Information Processing and Management, vol. 18, no. 6, pp. 289-293, 1982.

[50] M. J. Dutta-Bergman, "Complementarity in consumption of news types across traditional and new media," Journal of Broadcasting \& Electronic Media, vol. 48, no. 1, pp. 41-60, 2004.

[51] A. Nguyen and M. Western, "The complementary relationship between the internet and traditional mass media: The case of online news and information," Information research, vol. 11, no. 3, pp. 151-183, 2006.

[52] S. Finn, "Origins of media exposure linking personality traits to tv, radio, print, and film use," Communication research, vol. 24, no. 5, pp. 507-529, 1997.

[53] G. Gigerenzer and R. Selten, Bounded rationality: The adaptive toolbox. Mit Press, 2002.

[54] M. Mueller-Frank and M. M. Pai, "Social learning with costly search," American Economic Journal: Microeconomics, vol. 8, pp. 83-109, February 2016.
[55] D. L. McFadden and K. E. Train, "Consumers' Evaluation of New Products: Learning from Self and Others," Journal of Political Economy, vol. 104, no. 4, pp. 683-703, 1996.

[56] P. Singh and C. Phelps, "Networks, social influence and the choice among competing innovations: Insights from open source software licenses," Information Systems Research, vol. 24, no. 3, pp. 539-560, 2009.

[57] D. Bates, M. Mächler, B. Bolker, and S. Walker, "Fitting linear mixed-effects models using lme4," Journal of Statistical Software, vol. 67, no. 1, pp. 1-48, 2015.

[58] B. Li, P. V. Singh, and Q. Wang, "Zoom in ios clones: Examining the impact of copycat apps on original app downloads," 2014.

[59] E. Brynjolfsson, Y. J. Hu, and M. D. Smith, "From niches to riches: Anatomy of the long tail," Sloan Management Review, vol. 47, no. 4, pp. 67-71, 2006.

[60] E. Brynjolfsson, Y. Hu, and D. Simester, "Goodbye pareto principle, hello long tail: The effect of search costs on the concentration of product sales," Management Science, vol. 57, no. 8, pp. 1373-1386, 2011. 